\title{
PSEUDO-PERIOD OF DISTORTED LATTICES IN DISORDERED SOLIDS
}

\author{
M. A. GRADO-CAFFARO* and M. GRADO-CAFFARO
}

C/Julio Palacios 11, 9-B, 28029-Madrid (Spain)

(Received 17 January 1999; In final form 5 March 1999)

\begin{abstract}
Disordered lattices are discussed in terms of the spacing between adjacent atoms. This discussion is based on the randomness of the above spacing and on the crystalline potential by assuming a one-dimensional solid. Our theoretical considerations are useful to study certain aspects of amorphous semiconductors.
\end{abstract}

Keywords: Disordered lattices; spacing between adjacent atoms; amorphous semiconductors

\section{INTRODUCTION}

Analytical approaches to the study of disordered solids by using advanced tools of mathematical physics are really necessary since a considerable lack of knowledge on the above subject is due to the utilization of obscure techniques which are called by certain authors empirical techniques. As an example of mathematical-physics based methods for analyzing theoretically amorphous structures, we can mention Ref. [1]. In the same way as Ref. [1], we shall comment in the following certain aspects related to the randomness of the spacing between adjacent atoms in a distorted lattice; in particular, considerations upon crystalline potential will be made.

*Corresponding author. 


\section{THEORY}

First of all, we recall the following well-known fact: a solid in crystalline state presents a constant spacing between adjacent atoms; this spacing is the period of the corresponding crystalline lattice. In addition, it is also well-known that a given electron in the solid is submitted to a periodic potential so that the period of this potential is equal to the above spacing. The conception of this spacing as a random variable implies that the solid behaves as a disordered system. Then, for a disordered one-dimensional solid, we can write $V(x+s) \neq V(x)$, where $V$ denotes potential energy, $x$ is spatial coordinate (cartesian coordinate), and $s$ stands for lattice period. The parameter $s$ may be regarded as a random variable $[1,2]$. Now, by considering the mean value theorem, we have ( $s$ can be regarded as a pseudo-period):

$$
\begin{aligned}
V(x+s) & =V(x)+s\left[\frac{d}{d x}(V(x))\right]_{x \rightarrow x+\theta s} \\
& =V(x)-s F(x+\theta s) \quad\left(s_{1} \leq s \leq s_{2}\right)(0<\theta<1)
\end{aligned}
$$

but $F(x+\theta s)$ represents the magnitude of the force acting on a given electron submitted to a lattice field whose corresponding potential energy is $V$. On the other hand, we can do the following reasoning: since crystallinity involves periodicity of $V$ with a strictly fixed period, the magnitude of the quantity $s F(x+\theta s)$ constitutes a measure of disorder so that, if this magnitude is relatively small, we can claim that the system in question presents a state relatively close to the crystalline phase. Therefore, minimization of the above magnitude corresponds to a quasi-crystalline state.

Another alternative picture of the situation in question should consist of considering a first-order Taylor expansion of $V(x+s)$, namely:

$$
V(x+s) \approx V(x)-s F(x) \quad\left(s_{1} \leq s \leq s_{2}\right)
$$

However, the approximate formula (2) is only valid for relatively small $s$-values, that is to say, for $s \ll d$ where $d$ stands for the period of the crystalline lattice counterpart (see Ref. [1], p. 215). By virtue of this 
ref., we have:

$$
\int_{s_{1}}^{s_{2}} s x(s) d s=d
$$

where $x(s)$ is the probability density function of $s$ as a random variable. At this point, by taking into consideration the probability interpretation of standard quantum mechanics, $x$ is a random variable so $x+s$ is another random variable whose probability density function should be a useful tool for examining complex situations. Finally, we want to remark that, by taking into account expression (2), quasicrystalline systems correspond to a minimal $F(x)$ so that one has $V(x+s) \approx V(x)$, as expected.

\section{CONCLUDING REMARKS}

In the previous brief study, we have called $V$ the crystalline potential (potential energy corresponding to the crystalline state); in reality, this potential should be called potential of disordered lattice. In addition, we wish to emphasize that randomness is a crucial concept in our context so that complex situations in physics of amorphous materials can be studied by using special tools [1,3] which, to date, have not been employed in the current literature. In particular, statistical concepts and sophisticated methods of signal theory and theory of dynamical systems should be used in this context.

\section{References}

[1] Grado-Caffaro, M. A. and Grado-Caffaro, M. (1998). Act. Pass. Electronic Comp., 21, $213-215$

[2] Grado-Caffaro, M. A. and Grado-Caffaro, M. (1998).

[3] Franks, L. E., Signal Theory (Prentice-Hall, 1975). 

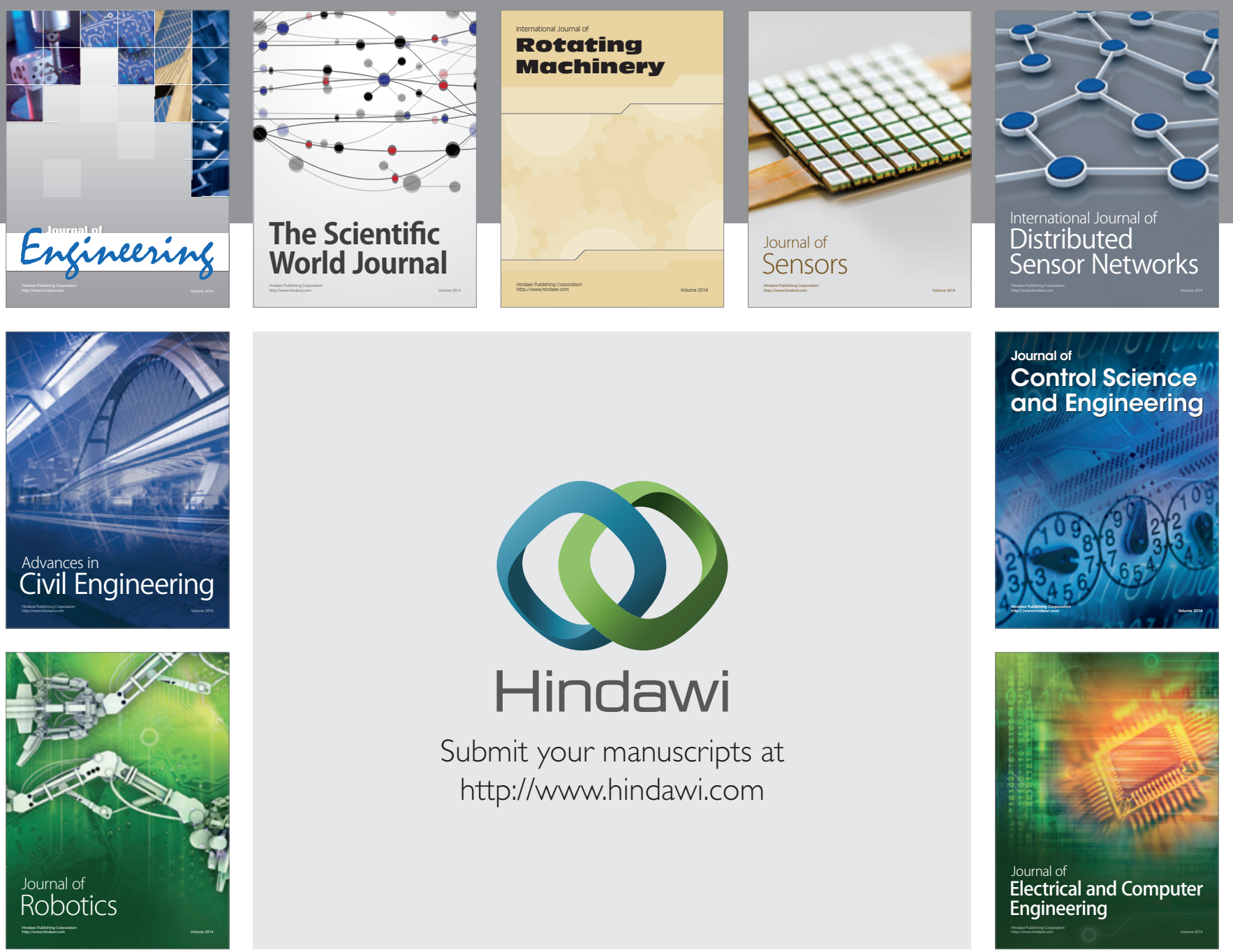

Submit your manuscripts at

http://www.hindawi.com
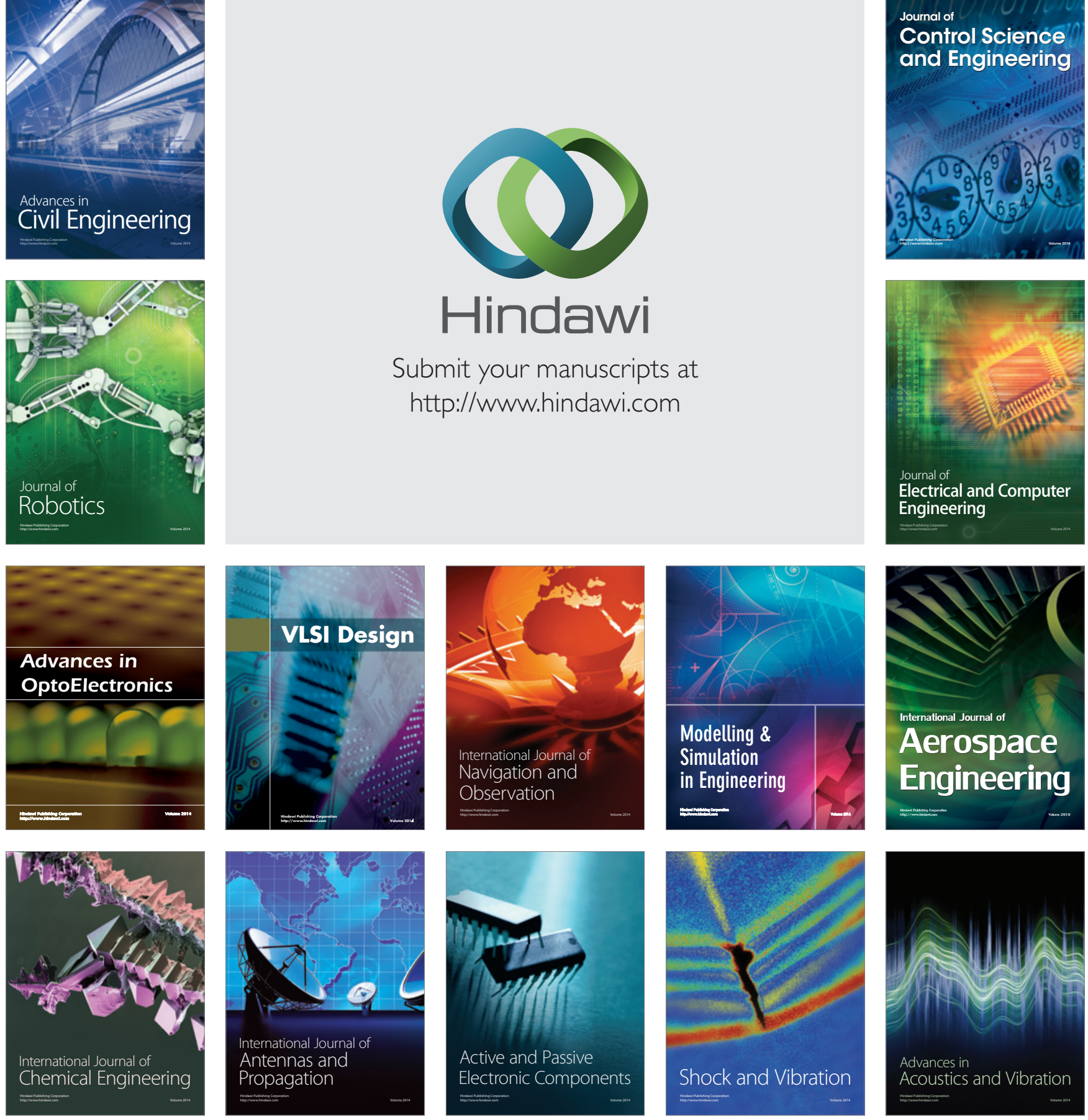